Gut, 1973, 14, 41-45

\title{
The effect of amino acids and dipeptides on sodium and water absorption in man
}

\author{
M. D. HELlieR, C. THIRUMAlai, AND C. D. HOLDSWORTH \\ From the Department of Medicine and Gastroenterology, St Bartholomew's Hospital, London, and \\ St Leonard's Hospital, London
}

SUMMARY A perfusion technique has been used to quantitate the effect of the amino acids glycine and alanine and the dipeptides glycyl-glycine and glycyl-L-alanine on sodium and water absorption from the human jejunum. Although no net absorption of sodium and water was seen in the absence of non-electrolytes, a significant stimulation occurred in the presence of both free amino acids and dipeptides. In the case of glycine and alanine, sodium and water absorption increased linearly with increasing concentration of the amino acid and saturation was not demonstrated over the range of concentrations studied. Molar ratios for net absorption of free amino acid and sodium were approximately 1:1 for all but the highest concentration of alanine. In contrast, the corresponding molar ratios for the two dipeptide solutions and sodium were approximately $1: 2$. Possible explanations for these observations are discussed.

There are important interactions between the absorption of sodium and certain non-electrolytes such as glucose and amino acids. These have been extensively studied in vitro, and the literature has been reviewed by Schultz and Curran (1970). The interaction is reciprocal. The presence of sodium in the incubation medium is necessary for the transport of sugars and amino acids. Conversely the transport of these non-electrolytes results in increased sodium transport.

In vivo the transport of glucose (Saltzman, Rector, and Fordtran, 1972) and of amino acid (Fleschler, Butt, and Wismar, 1966; Adibi, 1970) appears to be unaffected by the absence of sodium in the perfusing fluid, probably due to the failure to maintain a truly sodium-free state in the lumen. On the other hand, both glucose and the disaccharide maltose stimulate sodium and water absorption (Sladen and Dawson, 1969).

The effect of amino acids on sodium and water absorption has been less extensively studied in man and the effect of dipeptides has not hitherto been assessed. Adibi (1970) demonstrated stimulation of sodium and water absorption by leucine, but the effect of other amino acids on sodium and water absorption has not been studied. We have therefore

Received for publication 26 October 1972. measured the effect of the two neutral amino acids, glycine and alanine, on sodium and water absorption, and compared this with the effect of their dipeptides, glycyl-glycine and glycyl-alanine. As an example of a basic amino acid we chose arginine.

\section{Method}

All subjects studied were normal young adult male volunteers. Absorption was measured over a $30-\mathrm{cm}$ length of upper jejunum using a modified doublelumen tube incorporating a proximal occlusive balloon (Sladen and Dawson, 1970). Details of the method, and mode of calculation of the results, have been given elsewhere (Hellier, Perrett, and Holdsworth, 1970). All analytical methods with the exception of sodium determinations have been published previously (Hellier, Holdsworth, Perrett, and Thirumalai, 1972). Sodium was measured using a flame photometer (Evans Electroselenium Ltd (EEL) Clinical, 150).

Glycine and alanine were perfused at three different concentrations. Absorption of sodium and water from $10 \mathrm{mM}$ glycyl-glycine and $10 \mathrm{mM}$ glycylalanine was compared with that from $20 \mathrm{mM}$ glycine and a mixture of $10 \mathrm{mM}$ glycine and $10 \mathrm{mM}$ alanine respectively. Similar studies were carried out using arginine at concentrations of $10 \mathrm{mM}$ and $20 \mathrm{mM}$. 
Results

SALINE PERFUSION (FIG. 1)

The mean results in eight normal subjects show net secretion of both sodium and water into the lumen. This was evident to the perfused subjects who usually experienced discomfort and fluid diarrhoea.

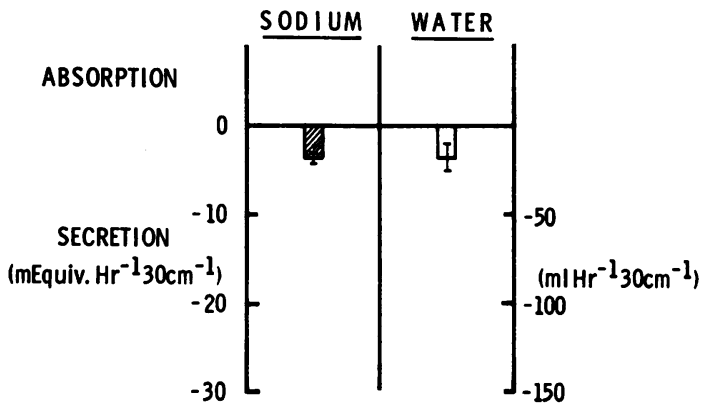

Fig. 1 Absorption of sodium and water by the jejunum during perfusion of isotonic saline. Results are the means of eight studies \pm 1 SEM.

NEUTRAL AMINO ACIDS (FIG. 2)

Both amino acids were readily absorbed and the absorption rates increased with increasing concentration. In the presence of these amino acids, there was significant and substantial enhancement of both sodium and water absorption at all concentrations studied. The abdominal discomfort and diarrhoea experienced during saline perfusions was diminished or abolished in the presence of these amino acids. With both glycine and alanine the stimulation of sodium absorption increased with increasing concentration of amino acid. In the case of glycine this increase was linear over the full range of concentration of infused amino acid, from $10 \mathrm{mM}$ to $50 \mathrm{mM}$,

\begin{tabular}{|c|c|c|c|}
\hline \multirow{2}{*}{$\begin{array}{l}\text { Concentration of } \\
\text { Alanine Infused } \\
(\boldsymbol{m M})\end{array}$} & \multirow{2}{*}{ 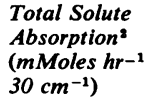 } & \multicolumn{2}{|c|}{ Water Absorption ${ }^{3}\left(\mathrm{ml} \mathrm{hr}^{-1} 30 \mathrm{~cm}^{-1}\right)$} \\
\hline & & Calculated & Observed \\
\hline $\begin{array}{l}10 \\
20 \\
50\end{array}$ & $\begin{array}{l}21 \cdot 2 \pm 5 \cdot 5 \\
34 \cdot 6 \pm 9 \cdot 2 \\
59 \cdot 6 \pm 3 \cdot 3\end{array}$ & $\begin{array}{r}71 \pm 18 \cdot 2 \\
115 \pm 34 \cdot 5 \\
199 \pm 40 \cdot 7\end{array}$ & $\begin{array}{r}83 \pm 33 \cdot 5 \\
119 \pm 28 \cdot 3 \\
219 \pm 30 \cdot 5\end{array}$ \\
\hline
\end{tabular}

Table Observed and calculated absorption of water during absorption of alanine ${ }^{1}$

${ }^{1}$ Results are expressed as means \pm 1 SEM

'Total solute absorption represents net absorption of $\mathrm{Na}^{+}, \mathrm{K}^{+}, \mathrm{Cl}^{-}$, and amino acid

${ }^{3} \mathrm{Calculated}$ on the basis that in isosmotic water absorption $3 \mathrm{mMoles}$ of solute are accompanied by $10 \mathrm{ml}$ of water
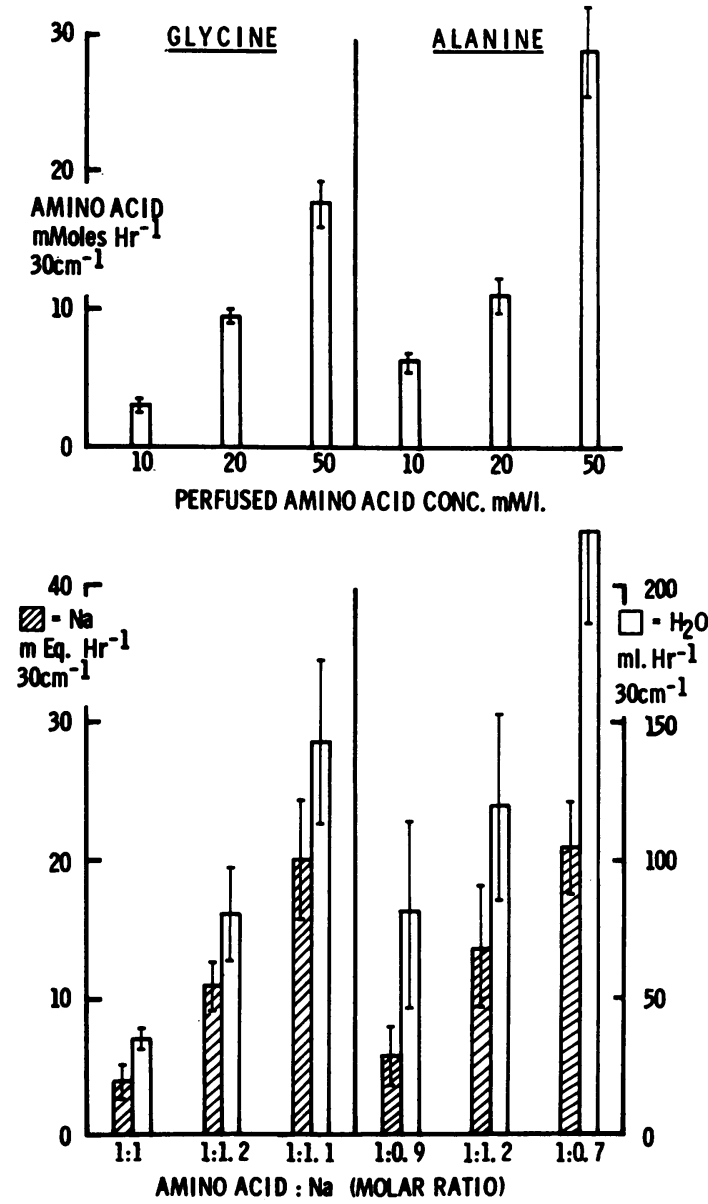

Fig. 2 Absorption of glycine and alanine by the jejunum, perfused at 10,20, and $50 \mathrm{mM}$. The corresponding data for sodium and water absorption from these solutions are shown in the lower half of the figure. Molar ratios for net transport of sodium and amino acids are indicated at the bottom of the figure for each solution studied.

Results are the means of six studies \pm 1 SEM.

and a 1:1 molar ratio between absorption of amino acid and sodium was maintained. In the case of alanine this 1:1 molar ratio was seen at $10 \mathrm{mM}$ and $20 \mathrm{mM}$ alanine, but at $50 \mathrm{mM}$ alanine the amino acid:sodium molar ratio was $1: 0 \cdot 7$ suggesting that the extent to which sodium absorption can be stimulated by this amino acid is limited.

Water absorption also increased with increasing concentration of amino acid. The apparent excess water absorption from $50 \mathrm{mM}$ alanine is due to the considerable increase in alanine absorption, and at 
all concentrations of both amino acids the measured water absorption could be accounted for as an osmotic effect secondary to net absorption of total solute. In the case of alanine this is illustrated in the Table.

ARGININE (FIG. 3)

In contrast to the findings with the neutral amino acids, arginine did not stimulate water absorption. At the higher concentration there was in fact net secretion of both sodium and water into the jejunal lumen.

Perfusion of high concentrations of arginine was not surprisingly associated with abdominal discomfort and watery diarrhoea.
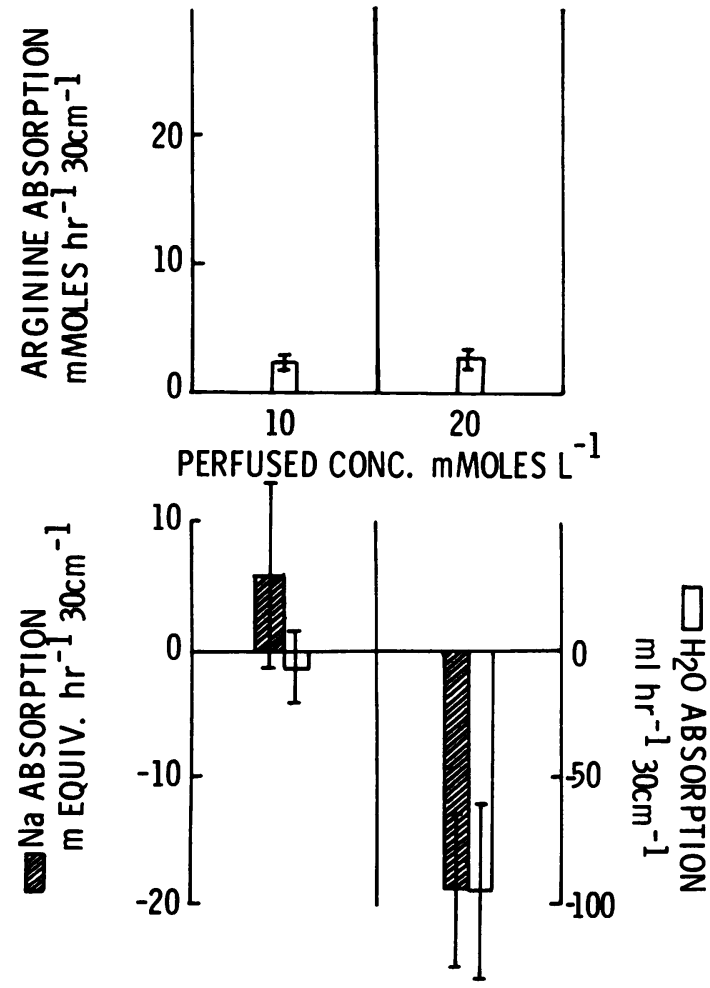

Fig. 3 The effect of arginine on sodium and water movement in the jejunum, when perfused at 10 and $20 \mathrm{mM}$. Results are the means of four studies \pm 1 SEM.

DIPEPTIDES (FIGS. 4 AND 5)

Both dipeptides significantly stimulated sodium and water absorption. In each case sodium and water absorption from the dipeptide solution was compared with that from a mixture of the constituent amino acids which was double the molar concentration and so contained the same number of amino acid molecules. For both dipeptides, the dipeptide:sodium molar ratio for absorbed solute was approximately 1:2 compared with $1: 1$ for the corresponding amino acid solution.
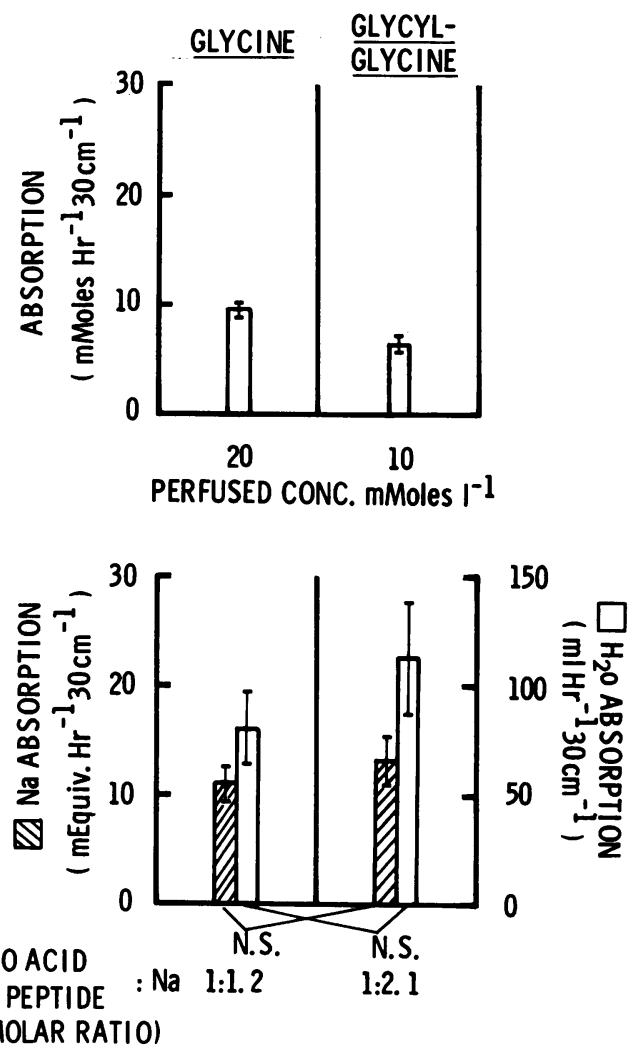

Fig. 4 Effect of 20 mMolar glycine and 10 mMolar glycyl-glycine on sodium and water absorption. Absorption of non-electrolyte is expressed in terms of $\mathrm{mMoles}$ of glycine or mMoles of glycyl-glycine. Corresponding data for sodium and water absorption are shown in the lower half of the figure. $P$ values are determined by Student's unpaired t test. Molar ratios for net sodium and amino acid or dipeptide absorption are shown at the bottom of the figure. Results are the means of six studies \pm 1 SEM.

\section{Discussion}

The use of an unmodified double lumen tube for the study of sodium and water absorption has been criticized, as infused fluid may reflux proximally or be diluted by fluid entering the test segment (Fordtran, 1969). When a proximal occluding balloon is incorporated in the system, absorption of glucose 

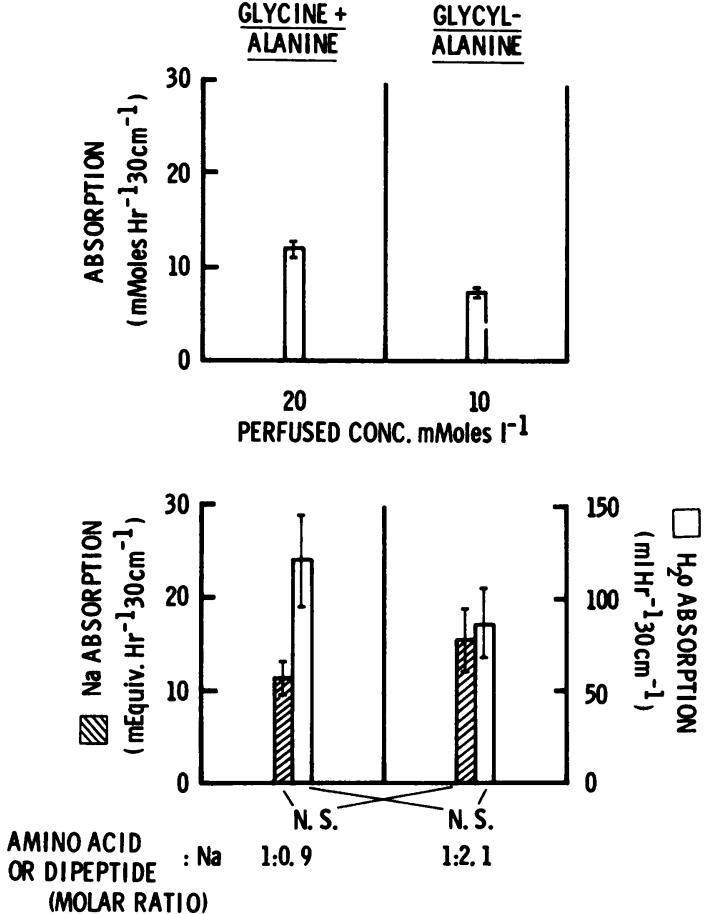

Fig. 5 Effect of a mixture of $10 \mathrm{mMolar}$ glycine and $10 \mathrm{mMolar}$ alanine compared with $10 \mathrm{mMolar}$ glycylalanine on sodium and water absorption. The data, together with $\boldsymbol{P}$ values and molar ratios, are presented as in Figure 4.

and water is higher with the balloon deflated than inflated (Modigliani and Bernier, 1971), suggesting that reflux of infused solution may indeed be significant. A proximal occluding balloon was therefore used in all our studies.

Our results using normal saline confirm previous observations that little or no sodium and water absorption occurs from this solution in the human jejunum (Sladen and Dawson, 1969). In our studies, there was in fact significant net secretion of both sodium and water, and, although Sladen and Dawson (1969) using no occlusive balloon found slight net absorption, this is probably due to the difference in technique.

The stimulation of sodium and water absorption by neutral amino acids was striking, and increased with increasing concentration of amino acid. Glucose has been shown to have a similar effect (Sladen and Dawson, 1969; Modigliani and Bernier, 1971), and this is not surprising as both glucose and amino acids stimulate sodium absorption in vitro (Schultz and Curran, 1970). In our studies sodium and water absorption increased with increasing concentration of amino acid, with evidence of saturation only at the highest concentration of alanine, and similar results were reported for glucose by Modigliani and Bernier (1971). The stimulation of sodium absorption in vitro by both glucose and amino acids is saturable and it may be that we would have found this at higher concentrations.

There is evidence in vitro that during absorption at the brush border amino acid and sodium are coupled in a 1:1 molar ratio (Schultz and Curran, 1970). This was claimed to be so for glucose and sodium absorption in man (Schedl and Clifton, 1963), but Sladen and Dawson (1969) found that this was an adventitious observation and the molar ratio varied at different concentrations. This is also evident from inspection of the date of Modigliani and Bernier (1971), studying the relationship between glucose and sodium absorption, and of Adibi (1970), studying the relationship between leucine and sodium absorption. In our own experiments the 1:1 molar ratio was observed at all three concentrations of glycine studied, and this ratio also obtained for two of the three concentrations of alanine. This almost constant molar ratio could be a coincidence, but is consistent with the in-vitro observation to which we have already referred.

Each of the two dipeptides studied also stimulated absorption of sodium and water. Evidence both in animals (Matthews, Craft, Geddes, Wise, and Hyde, 1968) and in man (Adibi, 1971; Hellier, Holdsworth, McColl, and Perrett, 1972) indicates that the initial step in absorption of these dipeptides is transport as intact dipeptides. As most dipeptides studied do not appear to enter the portal blood intact, it is likely that hydrolysis occurs within the mucosa. This, however, may not apply entirely in the case of glycyl-glycine where low concentrations of intact dipeptide have been detected in peripheral plasma during intestinal perfusion with high concentrations of glycyl-glycine (Adibi, 1971). The precise site of dipeptide hydrolysis is unknown. It could be at the brush border or deeper within the cytoplasm of the enterocyte. The stoichiometric relationship we observed between dipeptide and sodium was half that observed between the constituent amino acids and sodium. These are only net fluxes, and any conclusion drawn can only be tentative, but the data are consistent with the hypothesis that the dipeptide is hydrolysed before the interaction of the constituent amino acids with sodium. This would be analogous with the manner in which the disaccharide maltose stimulates sodium and water absorption (McMichael, Webb, and Dawson, 1967), although this is a less complex system, as disaccharides, unlike dipeptides, do not have independent 
transport systems. An alternative explanation of our data would be direct interaction between dipeptide transport and sodium transport on a molar basis differing from that seen between amino acid and sodium transport.

The findings for arginine contrast with those for the neutral amino acids. The failure to demonstrate an interaction with sodium and water, in particular the secretion of sodium and water seen at the higher concentration, suggests that arginine may have had a non-specific toxic effect upon the mucosa at these concentrations. However, it is of interest to note that in-vitro studies have shown that dibasic amino acid transport may occur independently of, or be only partly dependent upon, the presence of sodium (Christensen and Liang, 1966; Munck and Schultz, 1969).

The stimulation of sodium and water absorption by actively transported sugars, by amino acids, and by dipeptides may be of physiological importance in the absorption of both exogenous and endogenous fluid by the small intestine. It has found practical application in the treatment of cholera, where oral glucose reduces intravenous fluid requirements (Nalin, Cash, Islam, Molla, and Phillips, 1968) and a mixture of glucose and the amino acid glycine is even more effective (Nalin, Cash, Rahman, and Yunus, 1970). Many details of this relationship remain to be explored, including an extension of the concentration range of amino acids and dipeptides beyond those studied in this paper.

We should like to thank all those who volunteered to act as subjects for this study. We are grateful to the staff on the Metabolic Ward at St Bartholomew's Hospital and the Radiology Department for their helpful cooperation. Financial support for this work was received jointly from the Medical College of St Bartholomew's Hospital and the North East London Regional Hospital Board.

\section{References}

Adibi, S. A. (1970). Leucine absorption rate and net movements of sodium and water in human jejunum. J. appl. Physiol., 28, 753-757.

Adibi, S. A. (1971). Intestinal transport of dipeptides in man: relative importance of hydrolysis and intact absorption. J. clin. Invest., 50, 2266-2275.

Christensen, H. N., and Liang, M. (1966). Transport of diamino acids into the Ehrlich cell. J. biol. Chem., 241, 5542-5551.

Fleschler, B., Butt, J. H., and Wismar, J. D. (1966). Absorption of glycine and L-alanine by the human jejunum. J. clin. Invest., 45, 1433-1414.

Fordtran, J. S. (1969). Segmental perfusion techniques. Gastroenterology, 56, 987-989.

Hellier, M. D., Holdsworth, C. D., Perrett, D., and Thirumalai, C. (1972). Intestinal dipeptide transport in normal and cystinuric subjects. Clin. Sci., 43, 659-668.

Hellier, M. D., Holdsworth, C. D., McColl, I., and Perrett, D. (1972). Dipeptide absorption in man. Gut, 13, 965-969.

Hellier, M. D., Perrett, D., and Holdsworth, C. D. (1970). Dipeptide absorption in cystinuria. Brit. med. J., 4, 782-783.

Hydén, S. (1956). A turbidimetric method for the determination of higher polyethylene glycols in biological materials. $K$. LantbrHögsk. Anntr., 22, 139-145.

Matthews, D. M., Craft, I. L., Geddes, D. M., Wise, J., and Hyde, C. W. (1968). Absorption of glycine and glycine peptides from the small intestine of the rat. Clin. Sci., 35, 415-424.

McMichael, H. B., Webb, J., and Dawson, A. M. (1967). The absorption of maltose and lactose in man. Clin. Sci., 33, 135-145.

Modigliani, R., and Bernier, J. J. (1971). Absorption of glucose, sodium, and water by the human jejunum studied by intestinal perfusion with a proximal occluding balloon and at variable flow rates. Gut, 12, 184-193.

Munck, B. G., and Schultz, S. G. (1969). Lysine transport across the isolated rabbit ileum. J. gen. Physiol., 53, 157-182.

Nalin, D. R., Cash, R. A., Islam, R., Molla, M., and Phillips, R. A. (1968). Oral maintenance therapy for cholera in adults. Lancet, 2, 370-372.

Nalin, D. R., Cash, R. A., Rahman, M., and Yunus, M. D. (1970). Effect of glycine and glucose on sodium and water absorption in patients with cholera. Gut, 11, 768-772.

Saltzman, D. A., Rector, F. C., and Fordtran, J. S. (1972). The role of intraluminal sodium on glucose absorption in vivo. J. clin. Invest., 51, 876-885.

Schedl, H. P., and Clifton, J. A. (1963). Solute and water absorption by the human small intestine. Nature (Lond.), 199, 1264-1267.

Schultz, S. G., and Curran, P. F. (1970). Coupled transport of sodium and organic solutes. Physiol. Rev., 50, 637-718.

Sladen, G. E., and Dawson, A. M. (1969). Interrelationships between the absorption of glucose, sodium and water by the normal human jejunum. Clin. Sci., 36, 119-132.

Sladen, G. E., and Dawson, A. M. (1970). Further studies on the perfusion method for measuring intestinal absorption in man: The effects of a proximal occlusive balloon and a mixing segment. Gut, 11, 947-954. 